\title{
Nutritional and Physiological Significance of Luminal Glutamate-sensing in the Gastrointestinal Functions
}

\author{
Hisayuki UNEYAMA \\ Umami Wellness Research Group, Frontier Research Laboratories, Institute for Innovation, \\ Ajinomoto Co., Inc., 1-1 Suzuki-cho, Kawasaki-ku, Kawasaki 210-8681, Japan
}

(Received August 23, 2011)

\begin{abstract}
Recent evidence indicates that free amino acids are nutrients as well as acting as chemical transmitters within the gastrointestinal tract. Gut glutamate research is the most advanced among 20 amino acids. Free glutamate carries the umami taste sensation on the tongue and a visceral sensation in the gut, especially the stomach. In the field of taste physiology, the physiological meaning of the glutamate-derived chemical sense, the umami taste, has been proposed to be a marker of protein intake. Experimental evidence in gut glutamate physiology strongly supports this hypothesis. Free glutamate is sensed by the abdominal vagus and regulates gastrointestinal functions such as secretion and emptying to accelerate protein digestion. Clinical application of glutamate has also just begun to treat gastrointestinal disorders such as dyspepsia, ulcer, dry mouth and functional dyspepsia. In this review, we introduce recent advances in gut glutamate research and consider the possible contribution of glutamate to health.
\end{abstract}

Key words_-glutamate; umami taste; serotonin; taste receptor; gut nutrient sensing

\section{AMINO ACID-SENSING RECEPTORS DISTRIB- UTED IN THE TASTE AND GUT ORGANS}

During the past 20 years, a wide variety of amino acid-sensing receptors have been identified to distribute in non-neuronal peripheral tissues beyond the brain. For example, neuronal glutamate receptors such as metabotropic glutamate type 1 (mGluR1) and type 4 (mGluR4) are expressed in the taste cells on the tongue ${ }^{1-3)}$ as well as mucosal cells in the stomach and duodenum ${ }^{4,5)}$. Sweet taste receptors (T1R2/T1R3) and umami taste receptors (T1R1/ T1R3) for sensing amino acids on the tongue have also been indicated to be distributed in the intestine. ${ }^{6-10)}$ The calcium-sensing receptor (CaSR), which was originally discovered as a blood calcium sensor in the kidney, can also be activated by amino acids. ${ }^{11)}$ CaSR was reported to distribute in the gastrointestinal tract, including in the oral cavity. ${ }^{12)}$ Professor Hans Bräuner-Osborne recently reported that the GPRC6A receptor could act as lysine and arginine sensors. ${ }^{13)}$ This receptor is widely distributed in the gut organs, but the expression of GPRC 6A on taste tissue has not been identified.

e-mail: hisayuki_uneyama@ajinomoto.com

This Review is based on the content of Symposia 15

(S15) of the 131st Annual Meeting of the Pharmaceutical

Society of Japan.

\section{ROLE OF TASTE AND VISCERAL INFORMA- TION IN NUTRIENT HOMEOSTASIS}

The abdominal vagus is extensively distributed in the digestive tract from the esophagus to the upper gastrointestinal tract, and functions as the primary neuroanatomical substrate in the gut-brain axis to transmit meal-related signals in the gastrointestinal mucosa to the brain, which mediates neuronal gastrointestinal functions (e.g., food digestion, emptying and absorption) and conscious sensations (e.g., satiety, nausea, and discomfort) ${ }^{14-16)}$. Nutritional information from the gastrointestinal tract affects the regulation of food digestion and nutrient absorption as well as eating behavior, eventually contributing to the formation of dietary habits. A more detailed knowledge of the physiological significance of the gut nutrient perception would contribute to the development of new medicines to treat medical problems which have recently attracted a lot of attention, such as obesity, functional dyspepsia and irritable bowel syndrome, because these problems can also be called "eating habit style-related diseases."

Several lines of evidence suggest that visceral information mediated by vagal afferents play an important role in feeding behaviors. For example, rats fed an essential amino acid (lysine)-deficient diet showed appetite loss, lower growth and hypersensitivity to 
stressors. ${ }^{17)}$ In the normal diets, rats did not prefer the taste of lysine, due to its bitter taste. However, lysine-deficient rats showed a high preference for lysine solution because their palatability for it changed, due to the deficiency (Fig. 1(A)) . ${ }^{18)}$ Nerve recording studies of taste and vagal afferents showed that the lysine sensitivity of the taste afferents was not changed in rats who were lysine deficient but the sensitivity for vagal afferent sensitivity for lysine was dramatically increased up to one hundred times. ${ }^{19)}$ These researchers proposed the hypothesis that lysine deficiency changed the vagal afferent sensitivity for lysine, leading rats to selectively drink lysine-containing solution via brain coordination of taste and visceral information.

Regarding the preference for glutamate, an animal experiment has shown that a preference for the umami taste derived glutamate is somehow linked to the body's requirement to metabolize ingested dietary protein (Fig. 1(B)). In this experiment, rats received

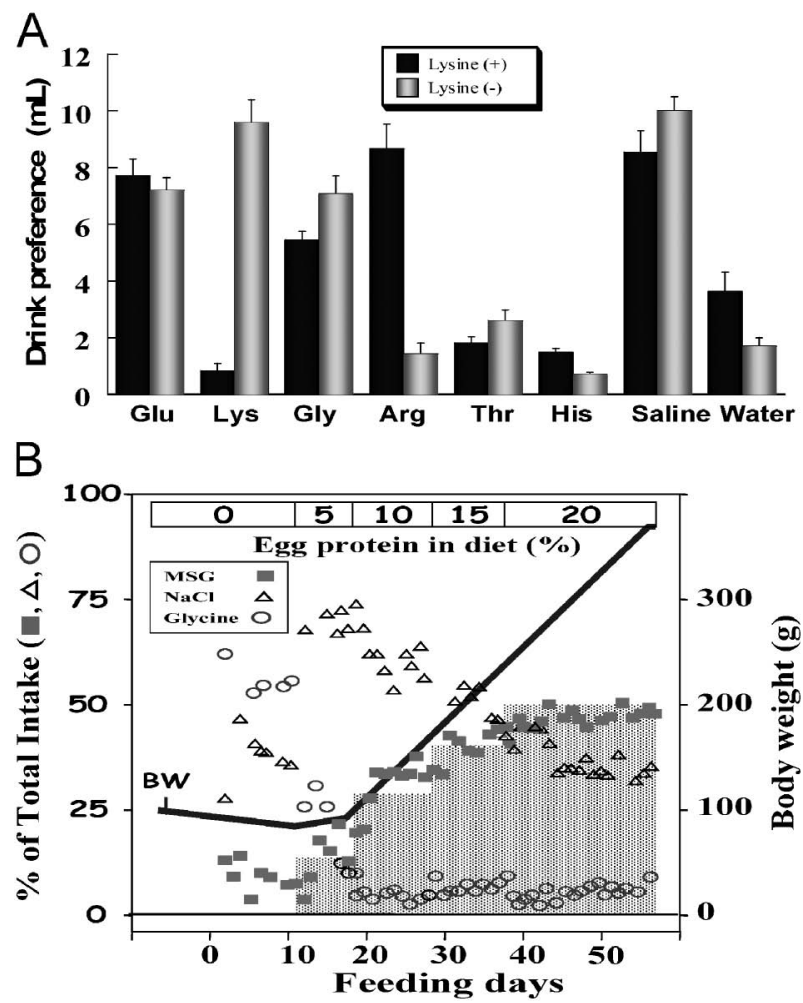

Fig. 1. Taste Preference and Body Nutrient Condition

A: Effect of lysine deficiency on taste preference in rats. Normal rats preferred umami (glutamate) and sweet (glycine) amino acids, but lysinedeficient rats also preferred the bitter (lysine) amino acid. Data is quoted from Tabuchi et al. ${ }^{18)}$ B: Relationship between protein intake and umami taste preference. Changes in taste preference for umami taste $(150 \mathrm{mM}$ monosodium glutamate; MSG), salty taste $(150 \mathrm{mM} \mathrm{NaCl})$ and sweet taste (500 $\mathrm{mM}$ Glycine) in rats fed with various contents of protein diets $(0-20$ $\%$ ). Data is quoted from Torii et al. ${ }^{19)}$ a diet containing stepwise increasing doses of purified egg protein for the indicated periods, and the preferences to three taste solutions $(\mathrm{NaCl}$, glycine and glutamate) were determined. When the protein content in diet is restricted to deficient levels around 0-5 $\%$, rats favor a salty taste $(\mathrm{NaCl}$ solution) and sweet taste (glycine solution) rather than the umami taste (monosodium glutamate solution). However, as the protein content in the diet increases, the taste preference for these solutions gradually changes. Interestingly, at a $20 \%$ protein diet, rats favor an umami solution rather than a $\mathrm{NaCl}$ solution or glycine solution. ${ }^{20,21)}$ This palatable change is explained by the requirement of glutamate as triggering molecules for gastric protein digestion. Thus, some lines of evidence strongly suggest that taste and visceral amino acid gut amino acid sensing can change feeding behavior to regulate the nutrient intake and help the maintenance of the body amino acid homeostasis.

\section{TASTE FUNCTIONS $V I A$ ORAL GLUTAMATE SENSING}

As previously described, the sense of taste is the chemical sense to intake essential nutrients, and the sense helps us to trigger oral digestion mastication with saliva as well as to prepare the gastric and pancreatic digestions via the cephalic phase response. ${ }^{22)}$ The sense of Umami taste occurs by the binding of glutamate to umami taste receptors expressed on the taste cells within taste buds on the tongue. Many candidate receptors for umami taste receptors have recently been revealed, such as G-protein coupled receptors (T1R1/T1R3 heterodimers, metabotropic glutamate 1 and 4 and their truncated receptors) ${ }^{23)}$

The sense of umami taste evokes a series of autonomic reflexes related to food ingestion. First, umami taste stimulation induces salivation, as observed in the other four basic tastes. It was reported that the potency in salivation of parotid glands in healthy volunteers is in the order of sour $>$ umami $>$ salty $>$ sweet $>$ bitter. ${ }^{24)}$ We studied the efficacy of the umami

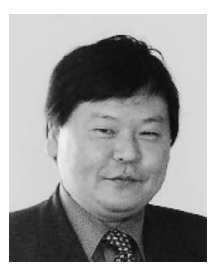

Hisayuki Uneyama
Associate General Manager and Group Manager. Umami Wellness Research Group, Forntier Research Labs., Institute for Innovation, Ajinomoto Co., Inc. 1989 Graduated from Tohoku University School of Pharmaceutical Sciences, 1989 Life Science Labs., Central Research Labs., Ajinomoto Co. Inc. 1998 Basic Research Labs., Central Research Labs. Ajinomoto Co. Inc. 2002 Chief Researcher, Institute for Life Sciences, Ajinomoto Co. Inc. Research fields: physiology of taste sense and body amino acid homeostasis. 
taste for inducing human whole saliva compared to that of the sour taste with healthy volunteers. The sour taste stimulation evoked a transient increase in salivation, but umami taste stimulation induced longlasting salivation of more than $10 \mathrm{~min}$. Interestingly, the total amount of saliva for $10 \mathrm{~min}$ with the umami taste was significantly larger than with the sour. ${ }^{25)}$ In contrast, there is no human evidence on the cephalic phase response induced by the umami taste, but it is estimated in animal experiments that the cephalic phase response with the umami taste can induce a cephalic phase response to activate vagal efferent activities of the gastric, pancreatic and celiac branches, and increase gastric and pancreatic exocrine (gastric and pancreatic juice) and endocrine (insulin) secretions. ${ }^{26,27)}$

\section{GLUTAMATE SIGNALING IN THE GUT}

Since Iggo et al. for the first time measured the nerve activity of the vagal afferent pathway with electrophysiological recordings, ${ }^{28)}$ a variety of nutrients such as glucose, amino acids and fatty acids have been found to activate the vagus from the gut, and a chemical perception system for nutrients has been assumed in the gastrointestinal mucosa, the so called "gut nutrient-sensing." For years, scientific knowledge about the mechanism and physiological sig- nificance of gut nutrient-sensing has not been advanced. However, identification of a series of amino acid-sensing receptors expressed in the gut as well as on the tongue elucidates the contribution of these receptors to the gut nutrient-sensing. Given this, what are the vagal sensing profiles for amino acids? I would like to introduce our recent research work. Niijima first reported that the injection of glutamate solution into the stomach, duodenum and portal vein increased nerve firings of afferent fibers of the gastric, celiac and hepato-portal vagal branches (Fig. $2(\mathrm{~A})) .{ }^{29)}$ He suggested that the chemical perception in those organs by the vagus may act as nutrient information to the brain. We also investigated the amino acid sensing profile by the vagus in the gastrointestinal tract.

The results are shown in Fig. 2(B). In the duodenum, all 20 amino acids could modulate the afferent activities of the celiac afferents. Glycine suppressed and glutamate, aspartate and tryptophan stimulate the afferent activities. ${ }^{30)}$ However, in the stomach, glutamate alone clearly increased the gastric afferent activities. ${ }^{31)}$ This strongly suggests that the intestinal mucosa has a mechanism that can recognize all the individual amino acids formed during protein digestion, but the gastric mucosa has a unique mechanism that can detect only free glutamate contaminated in
A)

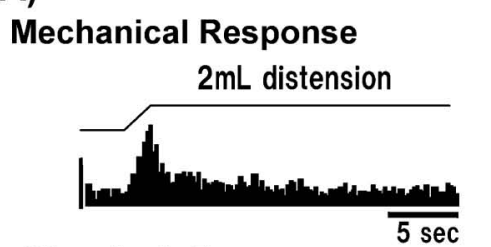

Chemical Response

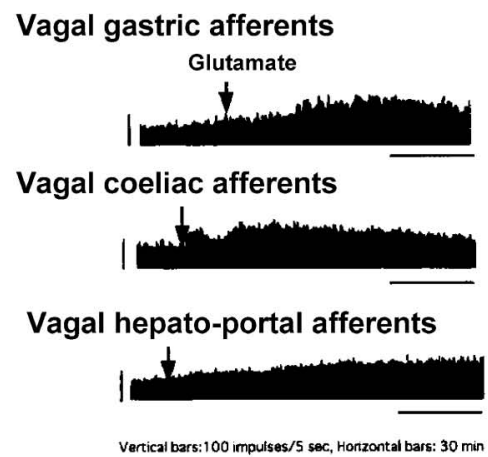

B)

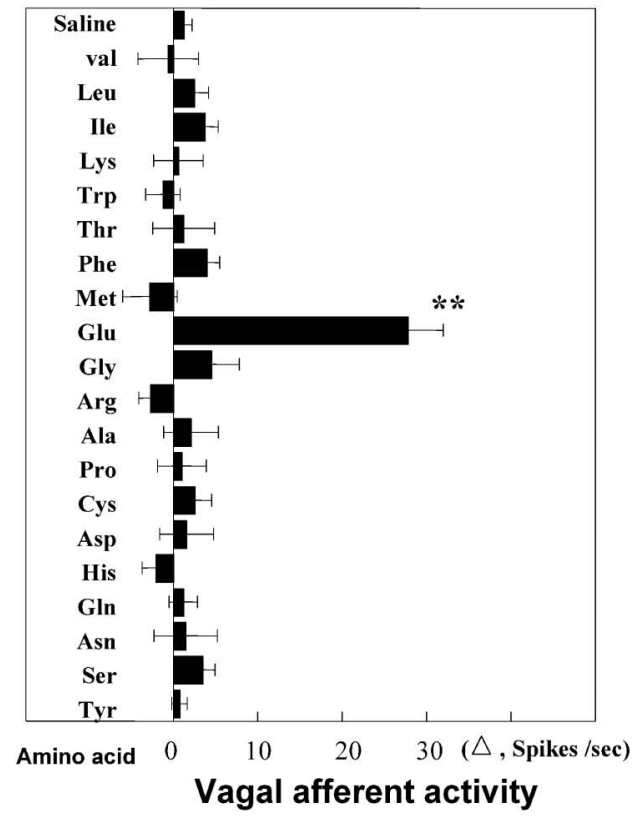

Fig. 2. Profiles of Amino Acid-sensing in the Rat Abdominal Vagus

A: Typical vagal afferent response of mechanical (gastric afferent) and glutamate (branches of abdominal vagus) in rats. B: Effects of 20 amino acids on the gastric vagal afferents. Each amino acid aqueous solution was introduced directly into the stomach at a concentration of $150 \mathrm{mmol} / \mathrm{l}$. Data are modified from Uneyama et al. ${ }^{31}$ 
proteins because the gastric proteolytic enzyme cannot digest protein to each amino acid.

Using the fMRI technique, we revealed that brain nuclei linked to feeding behaviors and gut functions are activated in response to the application of glutamate into the stomach. ${ }^{32-34)}$ Intra-gastric administration of $1 \%$ glutamate activated the brain nuclei including the dorsal vagal nucleus (DVN) and the nucleus of solitary tract (NTS), which is the output and input of the vagal nerve, and the insula cortex integrated with taste and visceral information. At the same time, each nucleus of the hypothalamus conveying feeding behavior and body temperature control was activated. Since the brain activation was diminished by cutting the vagus nerve, it was confirmed that most of the brain response induced by luminal glutamate was carried through the vagal afferent pathway (visceral sensation). On the other hand, intra-gastric application of another nutrient, glucose, brought the activation of some brain nucleus, but the activation was not affected by cutting the vagus nerve
(Fig. 3). Glucose-induced brain activation seems to be mediated by humoral factors (gut-pancreatic hormones). Interestingly, the brain mechanisms for gutnutrient sensing seem to vary depending on nutrients. We need to conduct further experiments to identify the brain-gut communication for individual nutrients.

What is the mechanism involved in the gastric glutamate sensing by the vagus? As an example of signal transduction cascade of gut amino acid-sensing, we examined gastric glutamate-sensing cascade within mucosa, using electrophysiological and pharmacological techniques (Fig. 4).35) Vagal afferent activation induced by luminal glutamate was abolished by the treatment with type 3 serotonin receptor inhibitor, granisetron, serotonin synthesis inhibitor, and pnitrophenylalanine (PCPA). This indicates that the glutamate response was mediated by serotonin. Moreover, the response was also abolished by the treatment with nitric oxidase inhibitor, L-NAME. In the case of gastric afferents, a NO donor, sodium nitroprusside, is known to activate the afferent fibers

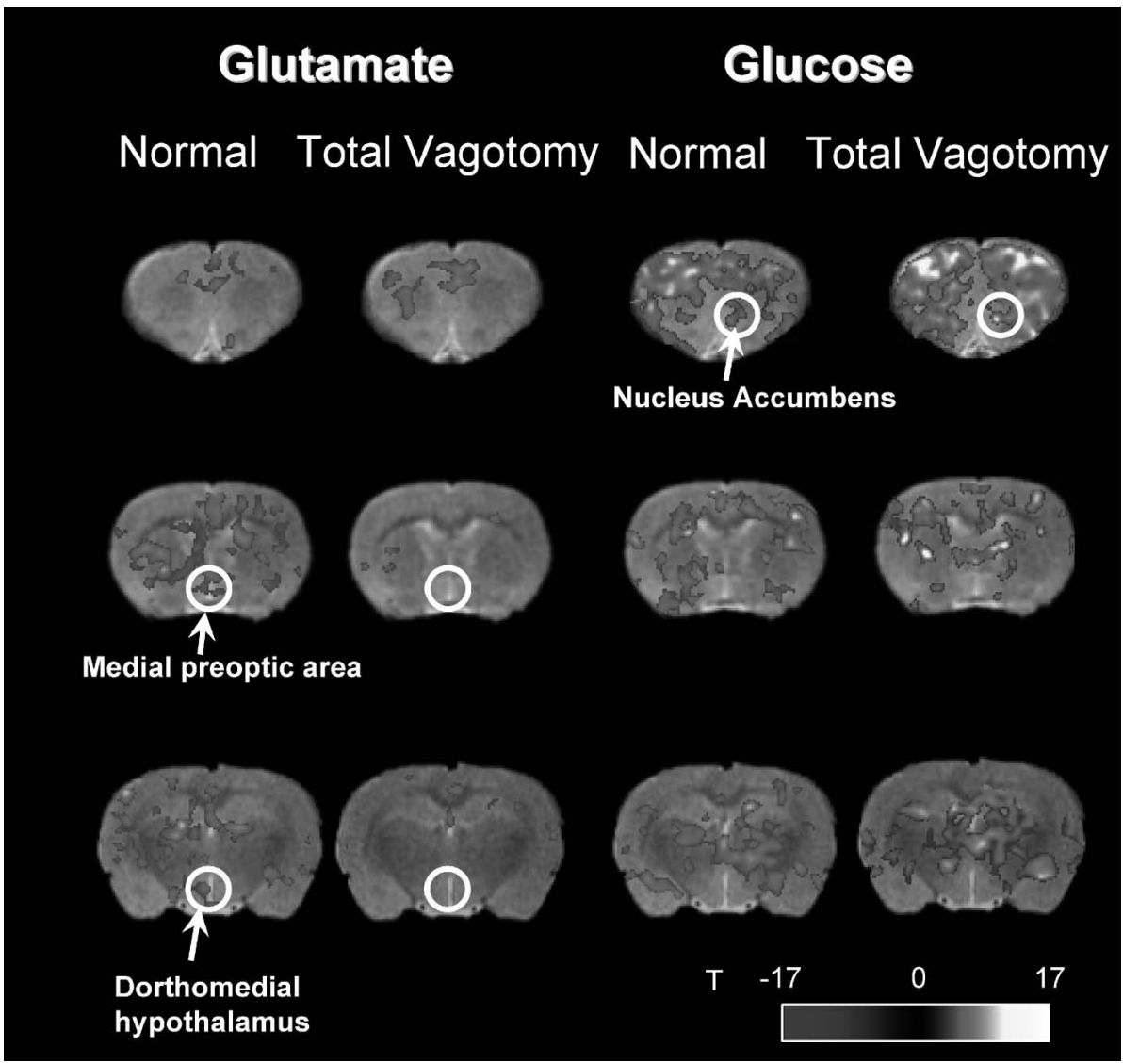

Fig. 3. Neuronal Pathways of Gut Glutamate and Glucose Perceptions within Rat Brain

fMRI image after intra-gastric administrations of glutamate and glucose. Almost all gastric glutamate-mediated signaling was diminished by the total vagotomy, but gastric glucose $(120 \mathrm{mmol} / \mathrm{l})$-mediated signaling was not affected by the vagotomy. The data are from Tsurugizawa et al. . $^{32}$ 

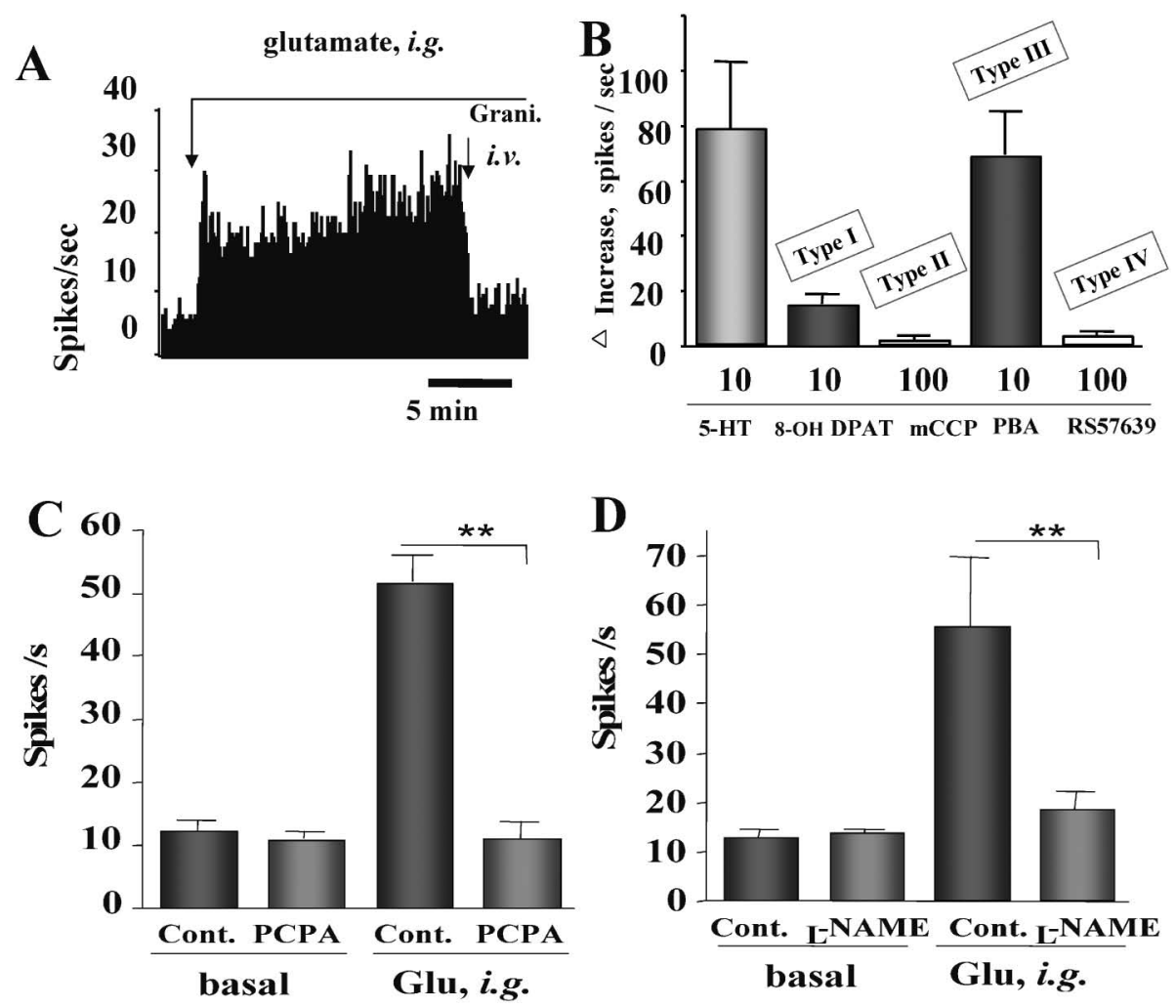

Fig. 4. Mechanisms of Luminal Glutamate-sensing in the Rat Gastric Mucosa

A: Effect of 5-HT3 antagonist, granisetron, on the gastric vagal excitation induced by luminal glutamate. B: 5-HT Profiles at the endings of gastric afferents. Each 5-HT agonist was intravenously applied (ug/kg., i.v.). C: Effect of mucosal 5-HT depletion on the glutamate responses. D: Effect of nonselective NOS blockade on the glutamate responses. Data are quoted from Uneyama et al. ${ }^{31}$ )

via the serotonin/5-HT3 cascade. ${ }^{36)}$ Taking these results together, it is indicated that luminal glutamate stimulates the mucosal receptors (perhaps mGluRs or T1Rs), which in turn stimulates mucosal NO/5-HT secretion couplings, and activates the 5-HT3 receptors at the nerve endings of gastric vagal afferents. However, we need further study to clarify whether this cascade is a common pathway in mucosal amino acid sensing or not.

Other experiments support the possibility that mucosal serotonin is involved in the gastric glutamate sensing. ${ }^{31)}$ In Fig. 5, the serotonin content within the gastric mucosa, and the contents of serotonin and its metabolite, 5-hydroxy acetic acid (5-HIAA) in the portal vein after $150 \mathrm{mmol} / \mathrm{l}$ glutamate was introduced into the stomach are shown. The data clearly indicate that the mucosal serotonin was increased but the blood serotonin never increased. 5-HT metabolites were increased in the portal vein. The mucosal serotonin might be mobilized and then metabolized within the mucosa to prevent the penetration of the bioactive substance, serotonin, directly into the bloodstream. In fact, gastric mucosa is known to have dense monoamine oxidases (MAOs). ${ }^{37,38)}$ Mucosal serotonin might be cleared by the enzyme or blood platelet uptake. Zhu et al. reported that glucose-sensing in the duodenum also mediated mucosal serotonin. ${ }^{39)}$ To date, more than $90 \%$ body serotonin is known to be localized within gastrointestinal mucosa, but its physiological role is unclear, despite involvement in emergency reactions such as vomiting and diarrhea. We think that the physiological role of gut mucosal serotonin is as a mediator of gut nutrient sensing. In Fig. 6, we summarize the possible mechanism involved in the gastric glutamate sensing.

\section{PHYSIOLOGICAL SIGNIFICANCE OF GLUTA- MATE-INDUCED VISCERAL INFORMATION}

We showed that gastric glutamate signaling potentiates gastric exocrine secretion such as gastric acid, pepsinogen and gastric juice, using Pavlov pouch dogs. In this experiment, we examined the effect of free glutamate on gastric acid secretion when injected 
A)
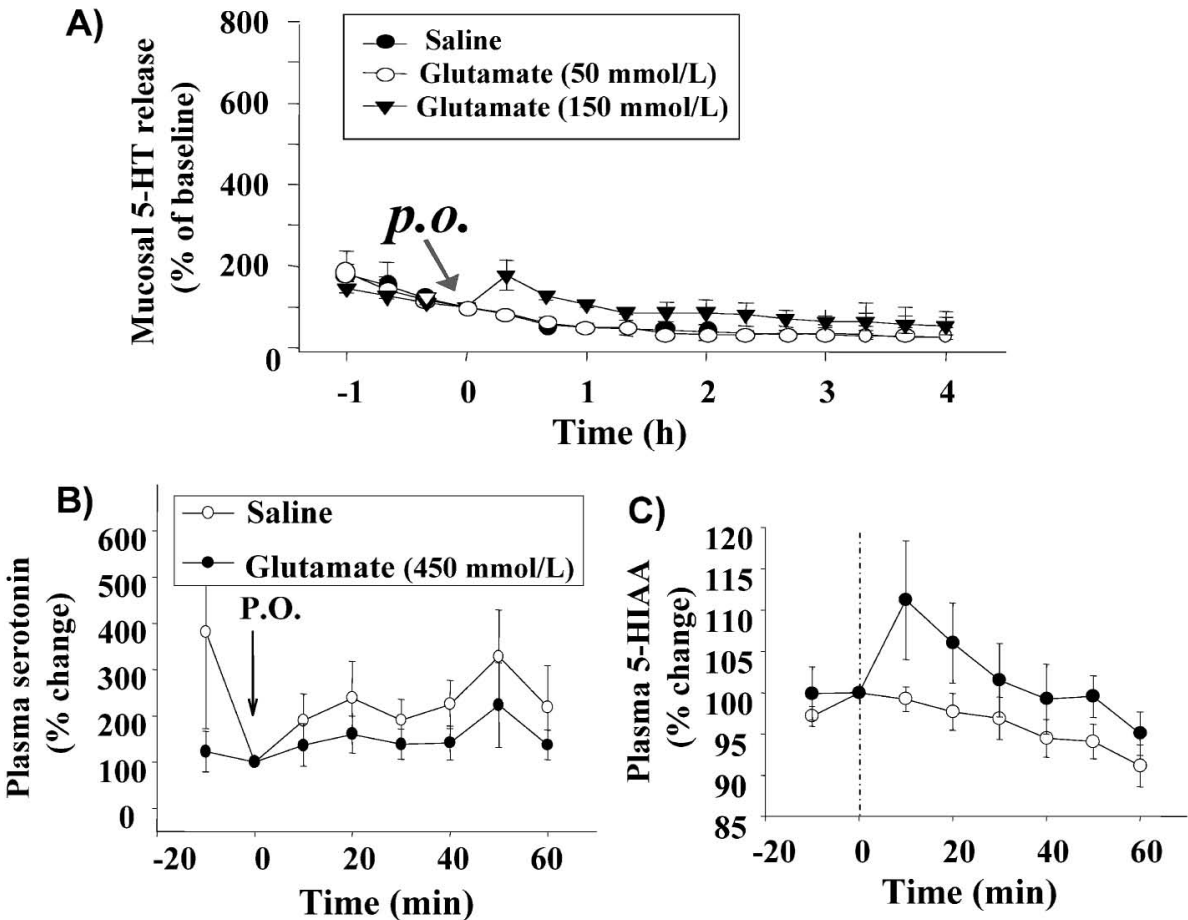

Fig. 5. 5-HT Secretion and Metabolism within Rat Gastric Mucosa

A: 5-HT release within the rat gastric mucosa in response to luminal glutamate loading. 5-HT was measured by the micordialysis technique. B: Portal plasma levels of 5-HT and 5-HAA after gastric glutamate loading. Data are quoted from Uneyama et al. $\left.{ }^{36}\right)$

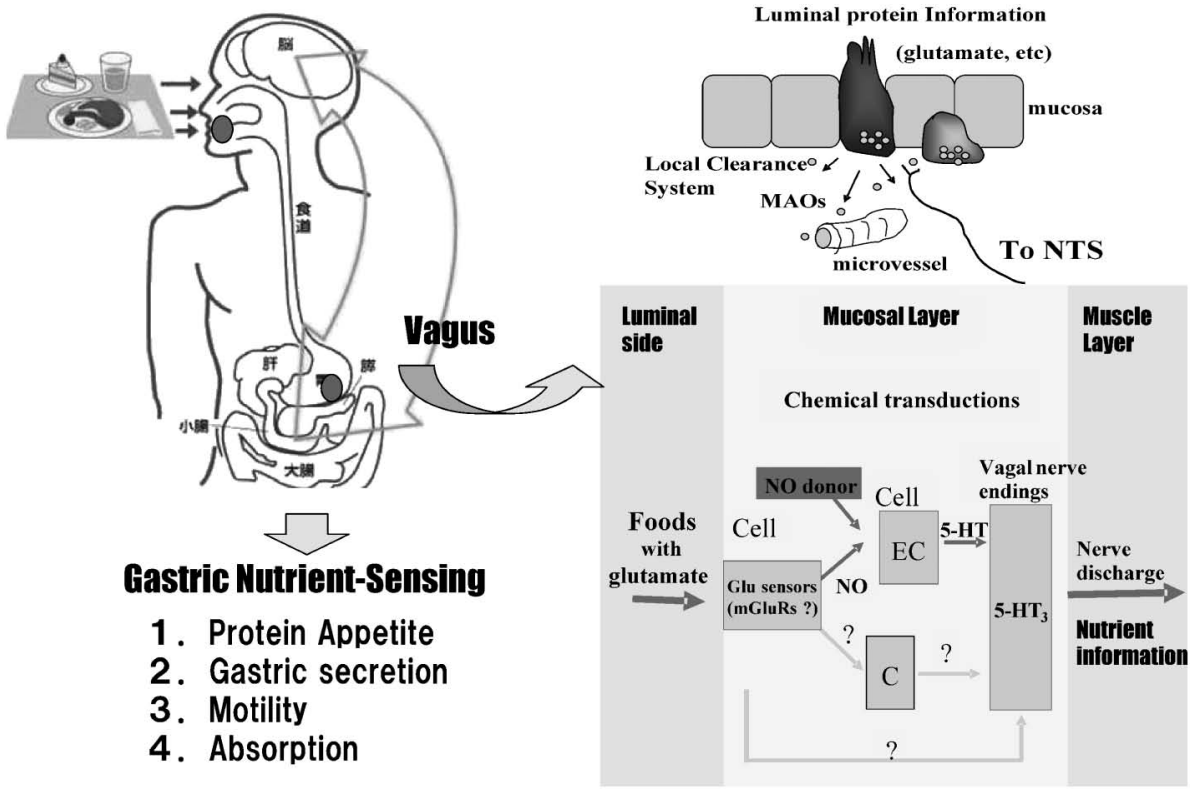

Fig. 6. Hypothesis of Gastric Glutamate Sensing

Luminal glutamate is sensed by the epithelial cells within gastric mucosa, leading to activation of the mucosal $\mathrm{NO} / 5$ - $\mathrm{HT}$ cascade, and finally activation of the 5-HT3 receptor at the nerve endings of gastric afferent fibers.

directly into the main stomach of Pavlov pouch dogs. ${ }^{40,41)}$ We used an enteral liquid diet (Elental ${ }^{\mathrm{TM}}$ ) as a nutrient, which is mainly composed of a mixture of amino acids, final nutrients digested from protein.
Elental $^{\mathrm{TM}}$ is composed of 17 amino acids (Ile, Leu, Lys, Met, Phe, Thr, Trp, Val, His, Arg, Ala, Asp, Gln, Gly, Pro, Ser, Tyr), vitamins, carbohydrates and micronutrients, and this amino acid diet com- 
pletely lacks glutamic acid in its composition. As a result, injection of the amino acid diet $(1 \mathrm{kcal} / \mathrm{ml}, 20$ $\mathrm{ml}$ ) into the main stomach could induce slight gastric secretion. Surprisingly, in the presence of the Elental $^{\mathrm{TM}}$ diet, intra-gastric application of free glutamate induced powerful gastric secretion in a dosedependent manner (Fig. 7). The intra-gastric administration of glutamate aqueous solution without Elental $^{\mathrm{TM}}$ failed to induce the gastric acid secretion at all. Interestingly, the glutamate effect seemed to appear specifically in the amino acid diet. Moreover, the gastric effects of glutamate markedly decreased by about $80 \%$ after cutting the vagus nerve innervations to the stomach. ${ }^{41)}$ That is, it was revealed in the dog experiments that glutamate directly acts in the stomach and helps the digestion of dietary protein in the stomach through stimulating vagal nerve dependent gastric exocrine secretion.

There is evidence in humans of food digestion via gut glutamate signaling. Dr. Zai and his colleague discovered that the addition of free glutamate to a high protein diet improved delayed gastric emptying and post-ingestive abdominal unpleasantness such as a heavy stomach (Fig. 8(A)).42) With a breath test using stable isotope-labeled $\left({ }^{13} \mathrm{C}\right)$ sodium acetate, they studied the supplemental effects of free glutamate to a high protein liquid diet (a mixture of $50 \%$ casein calcium and $50 \%$ dextrin, $1 \mathrm{kcal} / \mathrm{ml}, 400 \mathrm{kcal})$ and a 100 $\%$ pure dextrin diet $(1 \mathrm{kcal} / \mathrm{ml}, 400 \mathrm{kcal})$ on the human gastric emptying rate in 10 healthy adults. The addition of free glutamate $(0.5 \%)$ to the pure $100 \%$ dextrin diet failed to increase the gastric emptying rate, but the supplementation of free glutamate to the high protein diet significantly promoted the delayed gastric emptying. In the post-ingestive sensory evaluation study, free glutamate supplementation showed no effect on abdominal discomfort in young adults (between 20-39 years old), but in adults more than 40 years old, the post-ingestive abdominal discomfort such as a heavy stomach and fullness was much improved (Fig. 8(B)). ${ }^{43)}$ This result might suggest that the addition of free glutamate to a protein-rich diet

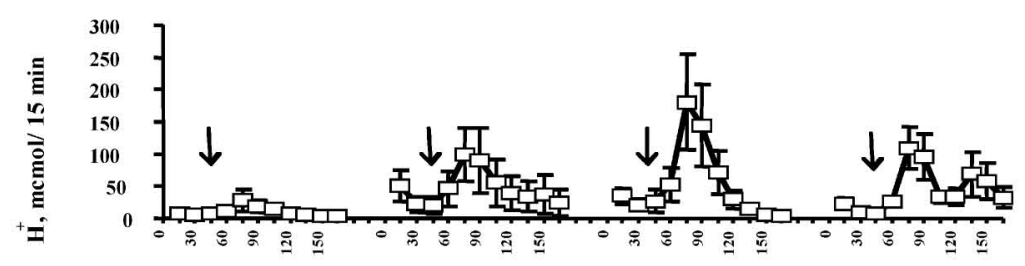

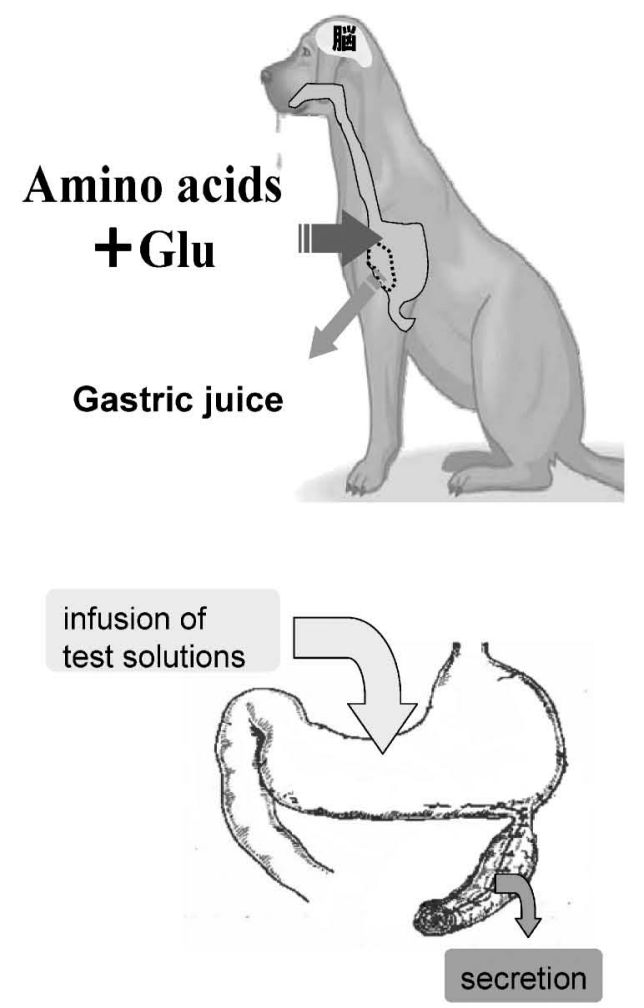

\section{Amino acids}
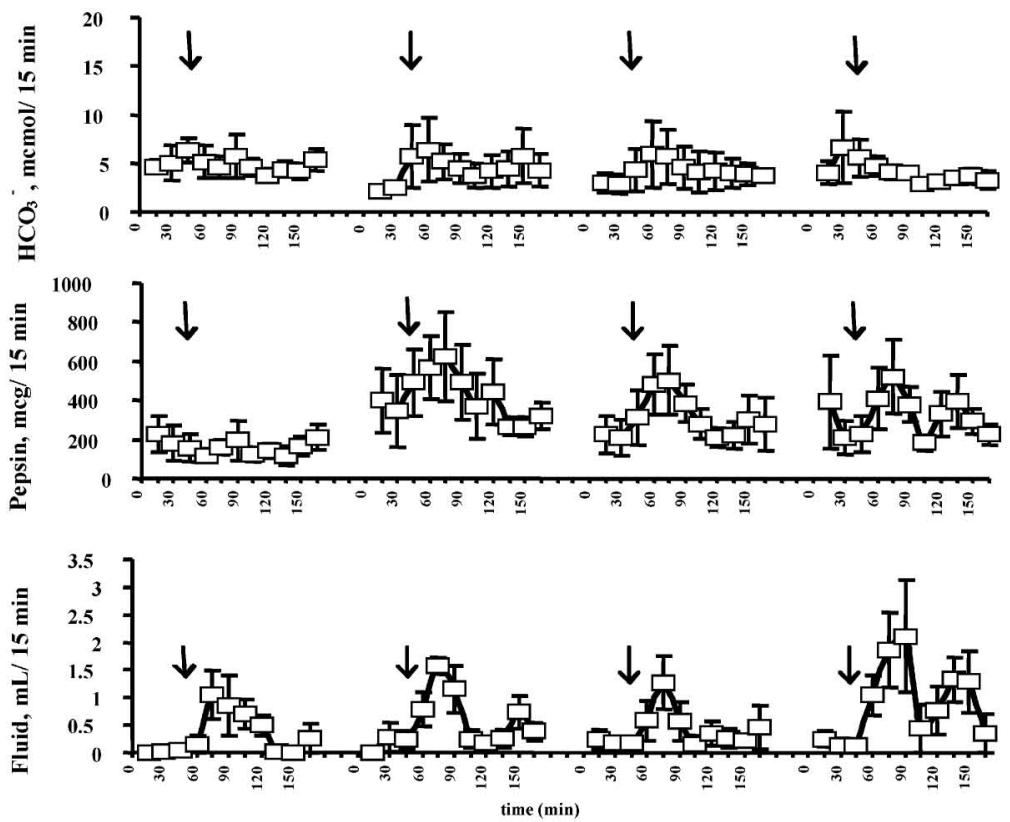

AA Diet

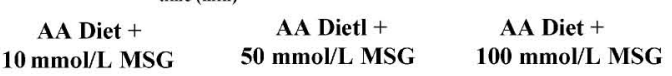

Fig. 7. Gastric Secretions Induced by Luminal Glutamate in Pavlov Pouch Dogs

Supplementation with 10-100 mmol/l monosodium glutamate enhanced gastric secretion induced by amino acid-rich diet (Elental) in Pavlov pouch dogs. Data are quoted from Uneyama et $a l_{.41}{ }^{41}$ 

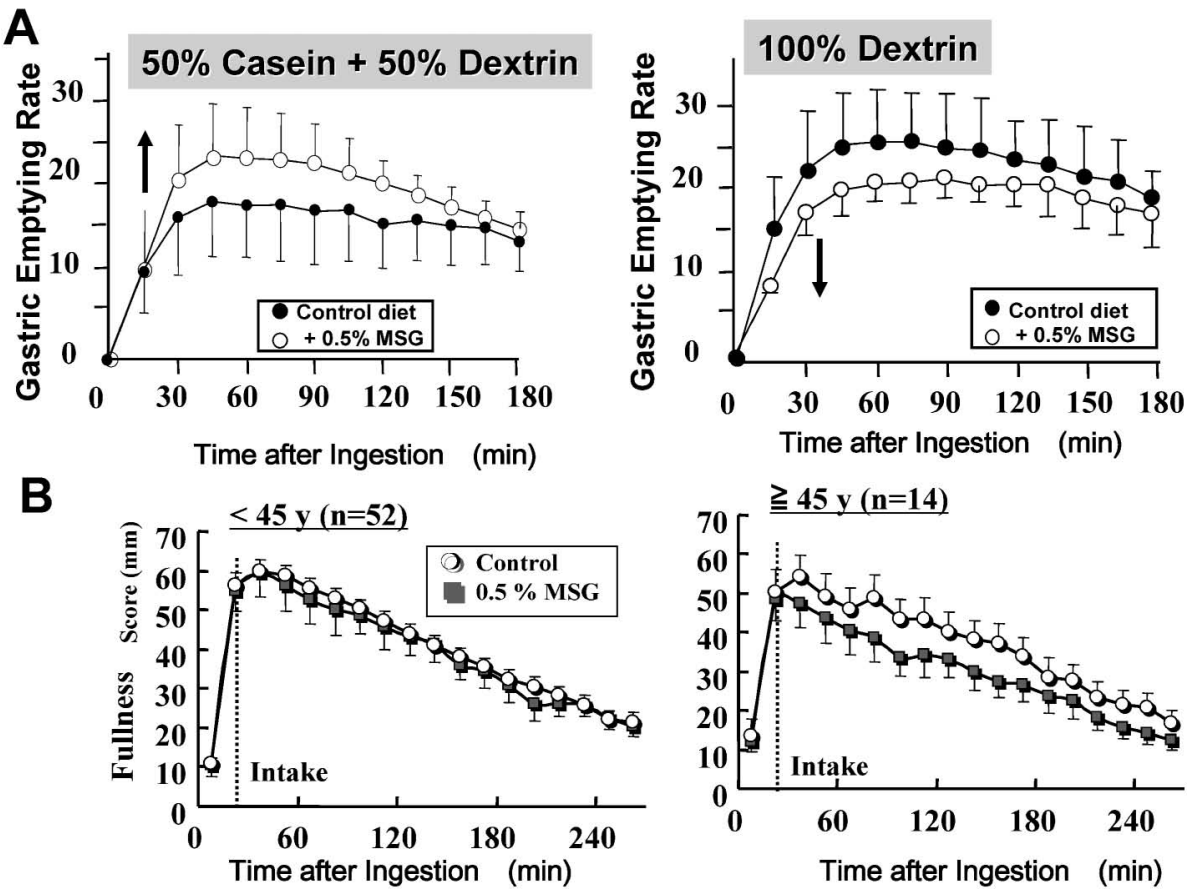

Fig. 8. Effects of Glutamate on Human Gastric Emptying and Post-ingestive Abdominal Feelings

A: Effects of glutamate supplementations to the protein-rich and pure dextrin liquid diets on the gastric emptying rates in healthy adult volunteers. Gastric emptying rate was measured by the breath test using a stable isotope $\left({ }^{13} \mathrm{C}\right.$ labeled acetate). $\mathrm{B}$ : Effect of glutamate supplementation to the protein-rich liquid diet on abdominal discomfort in healthy adult volunteers. Data are quoted. ${ }^{42,43)}$

enhanced gastric secretion, improving gastric protein digestion and accelerating the gastric emptying, leading to improvement of post-ingestive abdominal unpleasantness.

Hospitalized elderly often have advanced gastric atrophy with a decreased appetite and protein digestion, which cause a low-protein nutritional status with a plasma albumin level below $3.6 \mathrm{~g} / \mathrm{dl}$ (protein energy malnutrition: PEM). Based on the results measuring the free glutamate contents of typical hospital meals for the elderly, Toyama and Tomoe reported that the free glutamate intake of the hospitalized elderly was about half that of the average glutamate intake of healthy Japanese elderly.44) Therefore, to confirm the benefits of free glutamate intake in the hospitalized elderly, they conducted a clinical study of glutamate fortification to hospital meals. They obtained evidence that free glutamate supplementation to the daily main dish (porridge) of about $2.7 \mathrm{~g} /$ day improved elderly QOL measures such as the level of consciousness and some nutritional parameters (numbers of peripheral circulating lymphocytes and plasma albumin levels) . ${ }^{44-46)}$

\section{POSSIBLE CLINICAL APPLICATION AS A TARGET OF GUT GLUTAMATE SIGNALING}

Improvement in Enteral Nutrition Somekawa et al. reported that glutamate supplementation to an enteral liquid diet could prevent diarrhea induced by tube feedings in rats (Fig. 9) .47) Repetitive injection (bolus injection of $5 \mathrm{ml} /$ every $\mathrm{h}$ ) of purified protein liquid diet through a gastric tube could induce diarrhea after 7 injections. $0.5 \%$ free glutamate supplementation to this protein liquid diet could suppress the incidence of the diarrhea after the 7th repetitive injection. Moreover, taking advantage of glutamate use for a clinical diet, a clinical physician, Dr. Ohura, pointed out many benefits for nutritional management of enteral liquid diets containing $0.5 \%$ monosodium glutamate, which can reduce the incidence of diarrhea and gastro-esophageal reflux. ${ }^{48)}$ To date, the physiological importance of taste substances such as free glutamate (umami taste) contained in natural foods has been ignored, since historically, enteral liquid diets have been developed with the first priority of delivering simple purified nutrients to patients. We now believe that free glutamate supplementation to enteral liquid diets can improve the digestion, absorp- 


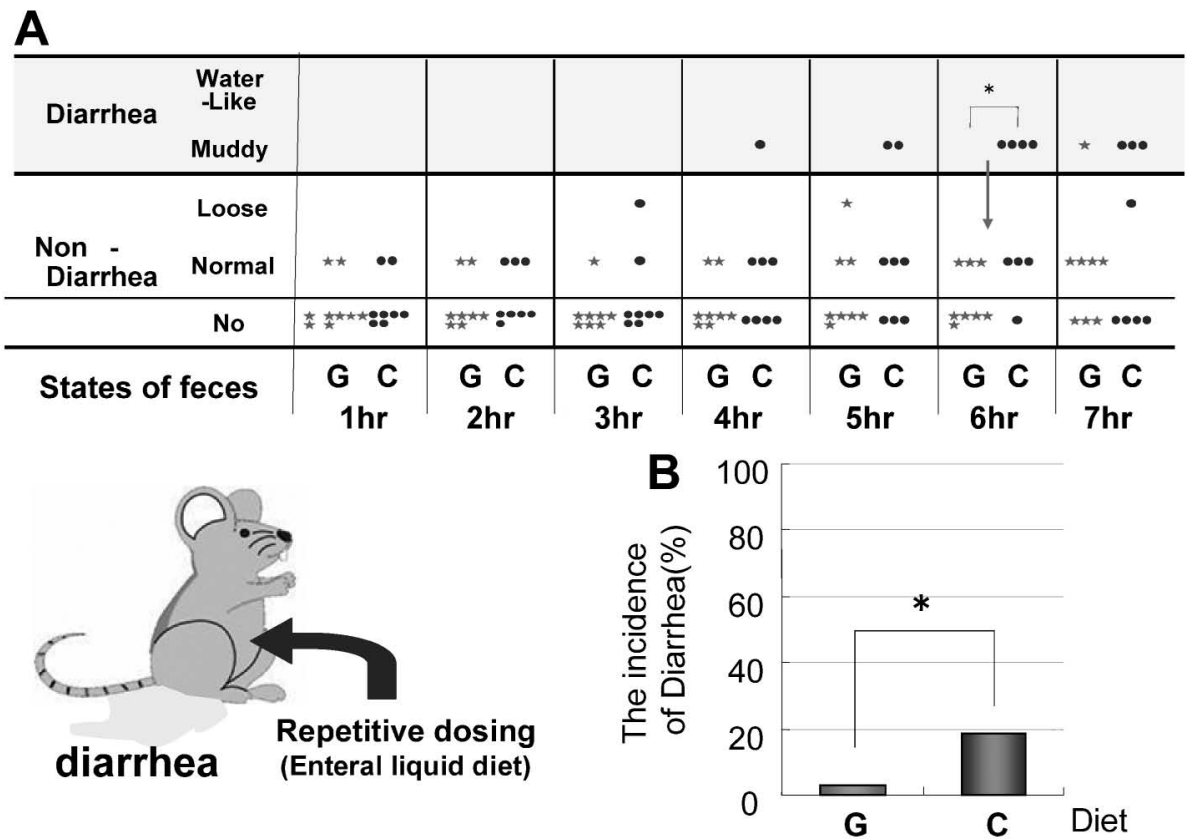

Fig. 9. Application of Glutamate for Improving Enteral Nutrition Therapy

A: Incidence of diarrhea induced by repetitive feedings through gastric tube in the glutamate fortified (G) and non-fortified (C) liquid diets. B: Effect of glutamate on total incidence of diarrhea $(7 \mathrm{~h})$. Data are modified from Somekawa et al. ${ }^{47)}$

tion and utilization of the diets through initiating visceral glutamate information from the stomach to the brain.

Dyspepsia Another report describing the possible contribution of free glutamate in protein digestion was reported from the Russian Academy of Sciences in 1992. ${ }^{49)}$ They studied the free glutamate fortification of hospital meals served to patients with chronic atrophic gastritis. Supplementation of about 2-3 grams of free glutamate per day for a month markedly improved basal acid output (BAO) and maximal acid output (MAO) and appetite in the hospitalized patients

Functional Dyspepsia Functional dyspepsia (FD) was defined as a clinical syndrome characterized by chronic or recurrent symptoms centered in the upper abdomen, in the absence of underlying histochemical disturbances. ${ }^{50)}$ The symptoms complex includes epi-gastric pain, bloating, postprandial fullness, early satiety, nausea, vomiting, belching and anorexia. Recently, it was reported that postprandial symptoms, especially postprandial fullness, is the most severe symptom in patients reporting aggravation by a meal. ${ }^{51)}$ There are three main possible mechanisms related to gastric disorders ${ }^{52)}: 1$ ) delayed gastric emptying, 2) impaired gastric accommodation to a meal, and 3) hypersensitivity to gastric disten- tion. Delayed gastric emptying was initially considered to be the main etiology of symptoms in FD. Therefore, Kusano et al. proposed the therapeutic possibility of glutamate in the FD patients by increasing gastric emptying. Delayed gastric emptying is a focal point of debate about anorexia caused by dyspepsia, and prokinetic agents are often administered in Japan for symptom therapy. ${ }^{53)}$

Gastrointestinal Ulcer Luminal glutamate induced mucosal secretions. Focusing on this glutamate effect, Professor Takeuchi proposed the possibility of preventing and treating drug-induced gastrointestinal ulcers. He clearly showed that glutamate-supplemented foods could protect non-steroid anti-inflammatory drug (NSAID) -induced gastric and duodenal ulcers in rat models. ${ }^{54)}$ The protective effects of glutamate were observed in $H$. pylori infected-gastric ulcers in the gerbil. ${ }^{55)}$

Oral care Saliva is essential for the maintenance of oral mucosa circumstances as well as oral functions such as speech, mastication, swallowing and taste sensation. Patients who visited hospitals with complaints of oral dryness in many cases have hypo-saliva secretion. ${ }^{56)}$ These patients also suffer from secondary symptoms such as difficulty speaking and swallowing, masticatory disturbance, mucosal pain and taste disorder. The patients' symptoms were 
relieved following increased salivation. Saliva also contains anti-bacteria, anti-viral and anti-fugal substances (i.e., IgA, defensins, mucin), and tissue recovery factors such as epidermal and transforming growth factors. Sever hypo-salivation after stroke and cancer radio and chemotherapy induced oral mucosa injury and bacterial outgrowth, leading to oral pain and aspiratory pneumonia. Sasano et al. attempted to treat dry mouth using umami taste stimulation instead of drug therapy (muscarine agonists), because umami taste induces long-lasting salivation from large salivary glands as well as minor salivary glands. ${ }^{57)}$

\section{REFERENCES}

1) Toyono T., Seta Y., Kataoka S., Kawano S., Shigemoto R., Toyoshima K., Cell Tissue Res., 313, 29-35 (2003).

2) Chaudhari N., Landin A. M., Roper S. D., Nat. Neurosci., 3, 113-119 (2000).

3) San Gabriel A., Uneyama H., Yoshie S., Torii K., Chem. Senses, 30, (Suppl. 1), i25-i26 (2005).

4) San Gabriel A. M., Maekawa T., Uneyama H., Yoshie S., Torii K., FEBS Lett., 581, 1119 -1123 (2007).

5) Akiba Y., Watanabe C., Mizumori M., Kauniz J. D., Gastrointest. Liver Physiol., 297, 781-791 (2009).

6) Jang H. J., Kokrashvili Z., Theodorakis M. J., Carlson O. D., Kim B. J., Zhou J., Kim H. H., Xu X., Chan S. L., Juhaszova M., Bernier M., Mosinger B., Margolskee R. F., Egan J. M., Proc. Nat. Acad. Sci. USA, 104, 1506915074 (2007).

7) Iwatsuki K., Nomura M., Shibata A., Ichikawa R., Enciso P. L., Wang L., Takayanagi R., Torii K., Uneyama H., Biochem. Biophys. Res. Commun., 402, 495-499 (2010).

8) Hass N., Schwarzenbacher K., Breer H., Cell Tissue Res., 339, 493-504 (2010).

9) Kokrashvili Z., Mosinger B., Margolskee R. F., Ann. N. Y. Acad. Sci., 1170, 91-94 (2009).

10) Mace O. J., Lister N., Morgan E., Shepherd E., Affleck J., Helliwell P., Bronk J. R., Kellett G. L., Meredith D., Boyd R., Pieri M., Bailey P. D., Pettcrew R., Foley D., J. Physiol., 587, 195-210 (2009).
11) Conigrave A. D., Brown E. M., Am. J. Physiol., 291, G753-761 (2006).

12) San Gabriel A., Uneyama H., Maekawa T., Torii K., Biochem. Biophys. Res. Commun., 378, 414-418 (2009).

13) Wellendorph P., Johansen L. D., Bräuner-Osborne H., Mol. Pharmacol., 76, 453-465 (2009).

14) Uneyama H., Tanaka T., Torii K., Nippon Yakurigaku Zasshi, 124, 210-218 (2004).

15) Berthould H., Regul. Pept., 149, 15-25 (2008)

16) Grundy D., Auton. Neurosci., 125, 76-80 (2006).

17) Smriga M., Kameishi M., Uneyama H., Torii K., J. Nutr., 132, 3744-3746 (2002).

18) Tabuchi E., Ono T., Nishijo H., Torii K., Physiol. Behav., 49, 951-964 (1991).

19) Torii K., Niijima A., Physiol. Behav., 72, 685 -690 (2001).

20) Nakamura E., Torii K., Uneyama H., Biol. Pharm. Bull., 31, 1841-1843 (2008).

21) Torii K., Mimura T., Yugari Y., "Biochemical Mechanism of Umami Taste Perception and Effect of Dietary Protein on the Taste Preference for Amino Acids and Sodium Chloride in Rats,' Marcel Dekker, Inc., New York, 1987.

22) Giduck S. A., Threatte R. M., Kare M. R., J. Nutr., 117, 1191-1196 (1987).

23) Chandrashekar J., Hoon M. A., Ryba N. J., Zuker C. S., Nature, 444, 289-294 (2006).

24) Hodson N., Linden R., Physiol. Behav., 89, 711-717 (2006).

25) Hayakawa Y., Kawai M., Torii K., Uneyama H., Jpn. J. Taste Smell Res., 15, 367-370 (2008).

26) Ohara I., Otsuka S., Yugari Y., Am. J. Physiol., 254, G424-428 (1988).

27) Niijima A., Togiyama T., Adachi A., Physiol. Behav., 48, 905-908 (1990).

28) Iggo A., Q. J. Exp. Physiol. Cogn. Med. Sci., 42, 398-409 (1957).

29) Nijima A., Physiol. Behav., 49, 1025-1028 (1991).

30) Uneyama H., Niijima A., San Gabriel A., Tanaka T., Torii K., Jpn. J. Taste Smell Res., 13, 411-414 (2006).

31) Uneyama H., Niijima A., San Gabriel A., Torii K., Am. J. Physiol., 291, G1163-G1170 
(2006) .

32) Tsurugizawa T., Uematsu A., Kondoh T., Nakamura E., Hasumura M., Hirota M., Uneyama H., Torii K., Gastroenterology, 137, 262-273 (2009).

33) Tsurugizawa T., Uematsu A., Uneyama H., Torii K., Neuroscience., 165, 244-251 (2009).

34) Uematsu A., Tsurugizawa T., Uneyama H., Torii K., Eur. J. Neurosci., 31, 1136-1143 (2010).

35) Uneyama H., Niijima A., Kitamura A., Torii K., Life Sci., 85, 782-787 (2009).

36) Uneyama H., Smriga M., Tanaka T., Torii K., Jpn. J. Taste Smell Res., 14, 453-456 (2007).

37) Okauchi H., Nakajima S., Tani T., Ito A., Arai R., Histochem. Cell Biol., 121, 181-188 (2004)

38) Fogel W. A., J. Neural. Transm., 32, (Suppl), 345-349 (1990).

39) Zhu J., Zhu X., Owyang C., Li Y., J. Physiol., 530, 431-442 (2001).

40) Zolotarev V. A., Khropycheva R., Uneyama H., Torii K., Ann. N. Y. Acad. Sci., 1170, 8790 (2009).

41) Uneyama H., Khropycheva R., Andreeva Y., Torii K., Zolotarev V. A., Jpn. J. Taste Smell Res., 16, 365-368 (2009).

42) Zai H., Kusano M., Hosaka H., Shimoyama Y., Nagoshi A., Maeda M., Kawamura O., Mori M., Am. J. Clin. Nutr., 89, 431-435 (2009)

43) Tanaka T., Fujita S., Kawai M., Okiyama A., Ogawa S., Hayakawa Y., Sakai M., Uneyama H., Torii K., Jpn. J. Taste Smell Res., 13, 415 -416 (2006).

44) Toyama K., Tomoe M., Inoue Y., Sanbe A., Yamamoto S., Biol. Pharm. Bull., 31, 1852 1854 (2008).

45) Tomoe M., Inoue Y., Sanbe A., Toyama K., Yamamoto S., Komatsu T., Ann. N. Y. Acad.
Sci., 1170, 82-86 (2009).

46) Yamamoto S., Tomoe M., Toyama K., Kawai M., Uneyama H., Am. J. Clin. Nutr., 90, 844S-849S (2009).

47) Somekawa S., Hayashi N., Niijima A., Uneyama H., Torii K., Br. J. Nutr., 24, 1-4 (2011).

48) Ohura N., Masuda M., Tanba M., Takeuchi H., Matsuda T., Komeda T., Ishida H., Saito H., Yamamoto S., Hirai K., J. JyomyakuKeicho Eiyo, 22, 345-352 (2007).

49) Kochetkov A. M., Shlygin G. K., Loranskaia T. I., Vasilevskaia L. S, Kondrashev S. Iu., Vopr Pitan, 5-6, 19-22 (1992).

50) Talley N. J., Stanghellini V., Heading R. C., Koch K. L., Malagelada J. R., Tytgat G. N., Gut, 45, 1137-1142 (1999).

51) Bisschops R., Karamanolis G., Arts J., Caenepeel P., Verbeke K., Janssens J., Tack J., Gut, 57, 1495-1503 (2008).

52) Sarnelli G., Caenepeel P., Geypens B., Janssens J., Tack J., Am. J. Gastroenterol., 98, 783-788 (2003).

53) Kusano M., Zai H., Hosaka H., Shimoyama Y., Nagoshi A., Maeda M., Kawamura O., Mori M., J. Pharmacol. Sci., 112, 33-36 (2010)

54) Amagas K., Ochi A., Kojo A., Mizunoe A., Taue M., Kinoshita N., Nakamura E. Takeuchi K., J. Pharmacol. Sci., (2011). (in press)

55) Nakamura E., Amagase K., Hasumura M., San Gabriel A., Uneyama H., Takeuchi K., Torii K., Ulcer Res., 36, 40-43 (2009).

56) Satoh-Kuriwada S., Shoji N., Kawai M., Uneyama H., Kaneta N., Sasano T., J. Health Sci., 55, 689-698 (2009).

57) Sasano T., Satoh-Kuriwada S., Shoji N., Sekine-Hayakawa Y., Kawai M., Uneyama H., Biol. Pharm. Bull., 33, 1791-1795 (2010). 